\title{
Emerging Role of Neurocognitive Sparing Radiotherapy: Can it be the New Standard of Care?
}

\author{
Swarupa Mitra ${ }^{1^{*}}$ and Sabeena Kataria ${ }^{2}$ \\ ${ }^{1}$ Department of Radiation Oncology, Rajiv Gandhi Cancer Institute and Research Centre, Rohini, India \\ ${ }^{2}$ Department of Radiation Oncology, St Stephans Hospital, India
}

"Corresponding author: Swarupa Mitra, Department of Radiation Oncology, Rajiv Gandhi Cancer Institute and Research Centre, Rohini, India, Tel: 919717041602; Email: swarupamitra@gmail.com

Received date: May 16, 2017; Accepted date: July 10, 2017; Published date: July 15, 2017

Copyright: (c) 2017 Mitra S, et al. This is an open-access article distributed under the terms of the Creative Commons Attribution License, which permits unrestricted use, distribution, and reproduction in any medium, provided the original author and source are credited.

\begin{abstract}
Whole-brain radiation therapy has been the standard form of treatment for advanced cancers, metastatic to the brain. It has the advantage of easy availability and delivery, and effectiveness in offering relief from symptoms for many suitable patients. Since most of these patients have poor outcomes and limited survival, oncologists in the past had a nihilistic approach and as a result, the potential toxicities emerging from WBRT have been largely neglected. With advances been made in neurosurgery, imaging, medical and radiation oncology, the results for many patients have improved significantly, particularly for those having favorable prognostic factors. Hence, the use of WBRT has come under scrutiny, due to its potential effect on the neurocognitive functions of the patient and their quality of life. This review article attempts to investigate if the various Neurocognitive sparing radiotherapy procedures can become a standard radiotherapy method for selected patients receiving whole brain radiotherapy for metastatic disease.
\end{abstract}

Keywords: Neurocognitive sparing radiotherapy; Radiation oncology; Brain tumor

\section{Introduction}

Patients with brain tumor, both primary and metastatic, constitute a large proportion of the patients receiving radiotherapy to the brain. Of the 1.4 million newly diagnosed tumors in the United States, around $30 \%$ would develop metastasis to the brain at some point of time, requiring Whole Brain Radiotherapy (WBRT) [1]. WBRT has proved to be an important mode of treatment for primary malignant brain tumors, tumors metastatic to the brain, childhood malignancies and as prophylactic cranial radiation in some hematologic malignancies [2]. WBRT for patients with secondaries in the brain has a long history.

It was used for the first time during the 1950s [3], when any tumors of the brain were thought to be associated with poor outcome and limited survival. But with improvements in oncologic treatments, patients with good prognostic factors have been seen to live longer. Hence, there is now a growing concern regarding neurocognitive toxicity after whole brain radiotherapy, especially in those patients with expected longer survival. WBRT prolongs survival, improves neurocognitive functions (NCF) but is paradoxically associated with decline in memory, especially recall and delayed recall [4].

Increased use of small field Radiotherapy like Stereotactic Radiosurgery (SRS) and Neurocognitive Sparing Radiotherapy (NSRT) have been used as an attempt to improve the quality of life of these patients by avoiding the neurocognitive domains of the brain.

\section{Cranial Effects of Whole Brain Radiotherapy}

The effects of radiation to the brain have been classified according to the time of occurrence after radiotherapy, as acute (within 6 weeks), delayed (up to 6 months) and late (after 6 months) [5]. The acute and the delayed effects may occur due to radiation induced cerebral oedema [6,7] and partly because of injury to the oligodendrocytes causing some disruptions in the synthesis of myelin. Unlike acute and delayed effects, the late effects are generally irreversible and progressive [8].

The pathophysiology of late effects is explained by the impact of radiotherapy on blood vessels of the brain or on the neuroglial cells and their precursor stem cells. The effect on blood vessels leads to ischemic events or bleeds years after WBRT, giving rise to the possibility of progressive deterioration of NCF [9].

The hippocampus in the brain is responsible for the formation of memory [10]. It is a structure, composed of the dentate gyrus and the cornu and is situated in the inner part of the temporal lobes, just lateral to the horns of the lateral ventricle.

It is a part of the limbic system, with its main function being learning, consolidation and retrieval of information and formation of new memories [11]. Radiation injury to the hippocampus, unilateral or bilateral is known to alter these functions, resulting in a decline in the ability of the patient to consolidate short-term with long-term memory [12].

There are several other factors like the simultaneous use of chemotherapy drugs, surgical intervention, use of other medications, comorbidities, baseline neurocognitive dysfunction that may affect the radiation effects to the normal brain parenchyma. Despite these confounding factors, several radiological studies have shown diffuse nonspecific changes in the white matter in all patients receiving WBRT to doses more than or equal to $20 \mathrm{Gray}(\mathrm{Gy})[13,14]$ (Figure 1). 


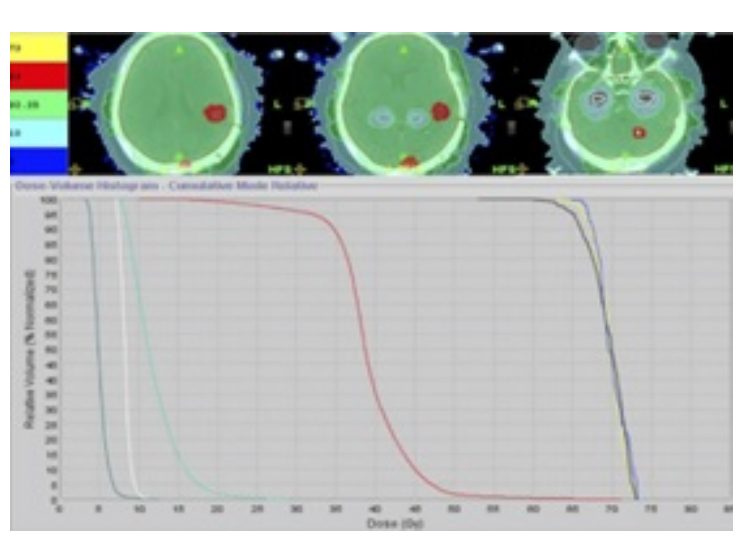

Figure 1: Planning for a typical hippocampus sparing radiotherapy.

\section{Hippocampal Avoidance}

Various methods have been employed to reduce the risk of post radiotherapy, neurocognitive decline in patients undergoing brain radiation. Memantine, which is an oral N-methyl-D-aspartate receptor antagonist and a neuro protector, has been used concurrently with WBRT. The RTOG 0614 trial, comparing WBRT with Memantine versus WBRT and a placebo showed that patients treated with Memantine had better cognitive function, with lesser decline in memory, and delayed time to decline in cognitive functions $[15,16]$ (Figure 2).

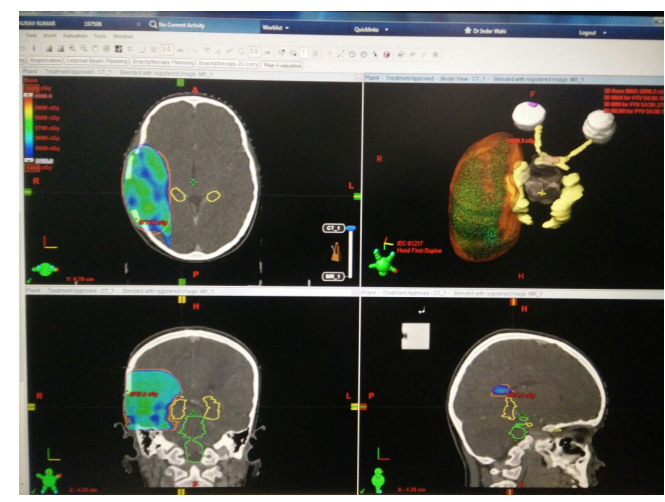

Figure 2: Neurocognitive sparing radiotherapy in paediatric glioma.

\section{Hippocampal Avoidance Radiation (HART)}

As established in various studies, radiation induced damage to neuronal progenitor cells in the sub granular zone of the hippocampi may result in a rapid decline in cognitive functions in patients [17].

Hence it has been proposed that the conformal sparing of the hippocampi during WBRT could result in preservation of NCFs $[18,19]$. Using modern radiotherapy planning systems, the mean dose of radiation to the neural stem-cell compartment of the hippocampus can be reduced by $80 \%$, while ensuring an adequate dose coverage and homogeneity in the rest of the brain [20]. It has also been seen that the incidence of recurrences within $5 \mathrm{~mm}$ of the hippocampi is very low.
Hence the sparing of the hippocampi may be considered safe as far as local recurrences are concerned [21]. Studies have shown that doses as low as even less than 10 Gy can cause neurocognitive dysfunction [22]. The central location of hippocampus makes it quite challenging to allow uniform dose delivery to the rest of the brain while avoiding the hippocampus. To combat this, various methods have been used, like MRI based contouring, conformal treatment with IMRT, volumetric arc radiotherapy (VMAT) and helical tomotherapy (HT). Among the various studies in Hippocampal sparing radiation therapy, Gondi et al reviewed five patients with recurrences in the brain and treated with WBRT where hippocampus and the avoidance region of $5 \mathrm{~mm}$ around it were contoured. They generated IMRT and HT plans for a prescribed dose of 30 Gy in 10 fractions and concluded that both techniques can produce a good hippocampal sparing with adequate target coverage and dose homogeneity. This pilot study was the basis of the phase II RTOG 0933 trial.

Blomstrand et al. [23] conducted a planning study using bilateral opposing fields, IMRT, Intensity Modulated Arc Therapy (IMAT) and Intensity Modulated Proton Therapy (IMPT) for irradiating medulloblastoma patients. The mean dose achieved to the hippocampus was $88.3 \%$ in IMAT, $77.1 \%$ in IMRT and $42.3 \%$ in IMPT. This study showed that chances of developing an impairment in memory after a prescribed dose of $23.4 \mathrm{~Gy}$ was estimated at $47 \%, 44 \%$, $41 \%, 33 \%$ with opposing fields, IMAT , IMRT and IMPT respectively [23]. Tarnawsky et al. in their study of HA WBRT in ten patients of lung cancer showed that even after decreasing the dose to NSC regions and maintaining the prescribed PTV coverage, both IMRT and VMAT were able to reduce the dose to NSC region by $45 \%$ and yet achieving the prescribed dose to the rest of the brain [24].

RTOG0933 was a single-arm phase II study comparing HA-WBRT for brain metastases with historical studies where patients have been treated with WBRT without hippocampal avoidance. Of 113 patients accrued, 42 could be evaluated at 4 months. Mean relative decline in Hopkins Verbal Learning Test-Revised (HVLT-R) Delayed Recall (DR) from baseline to 4 months was $7.0 \%$, which was significantly lower in comparison to the historical control $(\mathrm{P}<0.001)$. No decline in Quality of life (QOL) scores was observed. Two grade 3 toxicities and no grade 4 to 5 toxicities were reported. Median survival was 6.8 months. The trial concluded that it is possible to preserve memory and QOL by conformal avoidance of the hippocampus during WBRT [25]. In pediatric gliomas also significant reduction of both mean physical and mean biological equivalent doses were achieved respectively by $56 \%$ and $52.1 \%$ to the organs at risk (OAR) in comparison to plans in which these structures were not spared. The scope of Hippocampal avoidance radiotherapy has been studied in many situations NSCs compartments, the limbic structures and the whole hippocampus were contoured as OAR and included in the treatment plans by Marsh et al. [26] for evaluating hippocampal sparing radiotherapy plans in gliomas in children. Gutiérrez et al studied the feasibility of HA WBRT along with simultaneous integrated boost (SIB) for treatment of brain metastasis using helical tomotherapy [27].Recently there is a surge of interest to integrate Stereotactic radiosurgery with WBRT to improve local control. It is often possible to avoid hippocampus in both phases of treatment, SRS and WBRT. This approach has been compared to the WBRT+SIB and the latter has been found to be more effective in reducing doses to the hippocampus for patients with up to 8 metastases, as evidenced by Prokic et al. [28]. 


\section{Problems and Challenges Associated With the Use Of Hippocampal Avoidance Radiation}

Precise delineation of hippocampus to derive the neurocognitive benefit versus the risk of intracranial disease progression is of prime importance. Authors of the RTOG contouring atlas for hippocampal sparing prefer contouring only the subgranular zone as it is the main site where NSC niche is located. Moreover the need of IMRT to spare hippocampus owing to the central location within the brain without compromising the target coverage and homogeneity is likely to increase the financial burden as well as the time taken during treatment.

\section{Current Scenario}

Phase II RTOG 0933 trial has shown a moderate amount of effectiveness of HA WBRT but the use of the same in actual clinical practice seems doubtful. A survey was done among 1933 radiation oncologists of the US regarding the use of the modality, the reasons for using or not using the same. The result showed that $56 \%$ of the oncologists were not willing to employ HAWBRT based on a phase II trial result. And that majority of them were in favour of conducting a phase III trial for bringing HA WBRT into clinical practice [29].

\section{On-going Trials}

To validate the results of RTOG 0933, two large cooperative group trials have been planned to evaluate if HA decreases the risk for neurocognitive decline in patients undergoing WBRT. NRG (National Surgical Adjuvant Breast and Bowel Project, Radiation Therapy Oncology Group and Gynecologic Oncology Group) CC (Cancer Control) 003, is a randomized phase II/III trial of PCI comparing WBRT versus HA-WBRT in patients with both extensive and limitedstage small-cell lung cancer acquiring complete or partial response to chemotherapy. The second study, NRG-CC001, is a randomized phase III trial of HA-WBRT plus Memantine versus WBRT plus Memantine. Both these studies may help us to understand if HA-WBRT is worth the time and resources in today's era of value-based medicine.

\section{Conclusion}

The aim of this review is to summarize the rationale and the feasibility of sparing the hippocampus using various techniques for whole brain irradiation. Conventional WBRT are still the preferred modality of treatment for patients with multiple brain metastases and hippocampal sparing is generally not practiced outside clinical trials. Phase III studies are needed to understand not only the feasibility, but also the benefits of this modality and further implementation will depend on the results of these trials.

\section{References}

1. Siegel R, Ma J, Zou Z, Jemal A (2014) Cancer statistics. CA Cancer J Clin 64: 9-29.

2. Larson DA, Rubenstein JL, McDermott MW (2014) Devita, Hellman and Rosenberg's cancer. Principles and Practice of Oncology, p: 8.

3. DeVita VT, Lawrence TS, Rosenberg SA (2001) Philadelphia: Lippincott Williams \& Wilkins. Metastatic cancer to the brain, pp: 2461-2475.

4. JH Chao, R Phillips, JJ Nickson (1954) Roentgen-ray therapy of cerebral metastases. Cancer 7: 682-689.

5. Gondi V, Tomé WA, Mehta MP (2010) Why avoid the hippocampus? A comprehensive review. Radiother Oncol 97: 370-376.
6. Fink J, Born D, Chamberlain MC (2012) Radiation necrosis: Relevance with respect to treatment of primary and secondary brain tumors. Curr Neurol Neurosci Rep 12: 276-285.

7. Young DF, Posner JB, Chu F (1974) Rapid-course radiation therapy of cerebral metastases: results and complications. Cancer 34: 1069-1076.

8. Hindo WA, DeTrana FA, Lee MS (1970) Large dose increment irradiation in treatment of cerebral metastases. Cancer 26: 138-141.

9. Rider WD (1963) Radiation damages to the brain a new syndrome. Can Assoc Radiol J 14: 67-69.

10. Armstrong CL, Shera DM, Lustig RA (2012) Phase measurement of cognitive impairment specific to radiation therapy. Int J Radiat Oncol Biol Phys 83: e319-e324.

11. Scoville WB, Milner B (2000) Loss of recent memory after bilateral hippocampal lesions. J Neuropsychiatr Clin Neurosci 12: 103113.

12. Squire LR (1992) Memory and the hippocampus: A synthesis from findings with rats, monkey and humans. Psychol Rev 99: 195-231.

13. Monaco EA, Faraji AH, Berkowitz O, Parry PV, Hadelsberg U, et al. (2013) Leukoencephalopathy after whole brain radiation therapy plus radio surgery versus radio surgery alone for metastatic lung cancer. Cancer 119: 226-232.

14. Wara WM, Bauman GS, Sneed PK (1997) Brain stem and cerebellum. In : Principles and practice of radiation oncology, 3rd, Perez E, Brady LW (Eds), Lippincott-Raven, Phiadelphia, p: 799.

15. Day J, Zienius K, Gehring K, Grosshans D, Taphoorn M, et al. (2014) Interventions for preventing and ameliorating cognitive deficits in adults treated with cranial irradiation. Cochrane Database Syst Rev, p: 12.

16. Brown PD, Pugh S, Laack NN, Wefel JS, Khuntia D, et al. (2013) Radiation therapy oncology group. Memantine for the prevention of cognitive dysfunction in patients receiving whole-brain radiotherapy: A randomized, double-blind, placebo-controlled trial. Neuro Oncol 15: 1429-1437.

17. Abayomi OK (1996) Pathogenesis of irradiation-induced cognitive dysfunction. Acta Oncol 35: 659-663.

18. Chang JS, Perez-Andujar A, Barani IJ (2016) Estimating the probability of under dosing microscopic brain metastases with hippocampal-sparing whole-brain radiation. Radio Oncol 5: 30.

19. Truc G, Martin E, Mirjolet C (2013) The role of whole brain radiotherapy with hippocampal sparing. Cancer Radiother 17: 419-423.

20. Gondi V, Tolakanahalli R, Mehta MP (2010) Hippocampal-sparing whole- brain radiotherapy: A "how-to" technique using helical tomotherapy and linear accelerator-based intensity modulated radiotherapy. Int J Radiat Oncol Biol Phys 78: 1244-1252.

21. Ghia A, Tome WA, Thomas S (2007) Distribution of brain metastases in relation to the hippocampus: Implications for neurocognitive functional preservation. Int J RadiatOncolBiol Phys 68: 971-977.

22. Limoli CL, Giedzinski E, Rola R, Otsuka S, Palmer TD, et al. (2004) Radiation response of neural precursor cells: Linking cellular sensitivity to cell cycle checkpoints, apoptosis and oxidative stress. Radiat Res 161: 17-27.

23. Blomstrand M, Brodin NP, Rosenschöld PMA, Vogelius IR, Merino GS, et al. (2012) Estimated clinical benefit of protecting neurogenesis in the developing brain during radiation therapy for pediatric medulloblastoma. Neuro Oncol 14: 882-889.

24. Tarnawski R, Michalecki L, Blamek S, Hawrylewicz L, Piotrowski T, Slosarek K,et al. (2011) Feasibility of reducing the irradiation dose in regions of active neurogenesis for prophylactic cranial irradiation in patients with small-cell lung cancer. Neoplasma 58: 507-515.

25. Gondi V, Pugh SL, Tome WA, Caine C, Corn B, et al. (2014) Preservation of memory with conformal avoidance of the hippocampal neural stemcell compartment during whole-brain radiotherapy for brain metastases (RTOG0933): A phase II multi-institutional trial. J Clin Oncol 32: 3810-3816.

26. Marsh JC, Godbole R, Diaz A, Herskovic A, Turian J (2012) Feasibility of cognitive sparing approaches in children with intracranial tumors 
Citation: Mitra S, Kataria S (2017) Emerging Role of Neurocognitive Sparing Radiotherapy: Can it be the New Standard of Care?. J Neurol Neurophysiol 8: 435. doi:10.4172/2155-9562.1000435

Page 4 of 4

requiring partial brain radiotherapy: A dosimetric study using tomotherapy. J Cancer Ther Res 1: 1.

27. Gutiérrez AN, Westerly DC, Tomé WA, Jaradat HA, Mackie TR, et al. (2007) Whole brain radiotherapy with hippocampal avoidance and simultaneously integrated brain metastases boost: A planning study. Int $J$ Radiat Oncol Biol Phys 69: 589-597.

28. Prokic V, Wiedenmann N, Fels F, Schmucker M, Nieder C, et al. (2013) Whole brain irradiation with hippocampal sparing and dose escalation on multiple brain metastases: A planning study on treatment concepts. Int J Radiat Oncol Biol Phys 85: 264-270.

29. Slade AN, Stanic S (2016) The impact of RTOG 0614 and RTOG 0933 trials in routine clinical practice: The US survey of utilization of memantine and IMRT planning for hippocampus sparing in patients receiving whole brain radiotherapy for brain metastases. Contemp Clin Trials 47: 74-77. 